\title{
Synthesis and Characterization of Magnetic-Optical Co-Au Core-Shell Nanoparticles
}

\author{
Yuping Bao, ${ }^{\dagger, \S}$ Hector Calderon, $\$$ and Kannan M. Krishnan $*, \dagger$ \\ Materials Science and Engineering, University of Washington, Seattle, Washington 98195-2120, and \\ Departamento Ciencia de Materiales, ESFM-IPN, Mexico
}

Received: October 19, 2006; In Final Form: November 27, 2006

\begin{abstract}
We report the successful synthesis of $\mathrm{Co}-\mathrm{Au}$ core-shell nanoparticles by reducing an organo-gold compound onto cobalt seeds with a weak reducer in a nonpolar solvent. The core-shell morphology was unequivocally confirmed by complementary structural, magnetic, and optical property measurements. High-resolution transmission electron microscopy and Z-contrast imaging shows a gold shell composed of multiple grains. Electron-energy loss spectroscopy confirms the chemically distinct characteristics of the core (Co) and shell $\mathrm{Au})$. Temperature-dependent magnetic property measurements confirm that the particles are superparamagnetic with a blocking temperature, $T_{\mathrm{B}} \sim 55 \mathrm{~K}$, consistent with a magnetic diameter $\sim 6 \mathrm{~nm}$. The UV-visible absorption spectra of these nanoparticles show a red shift (relative to pure gold nanoparticles) in agreement with a Au-shell morphology.
\end{abstract}

\section{Introduction}

Nanoscale core-shell structures have received considerable attention recently because, in principle, their physical and chemical properties can be tuned by controlling their chemical composition and the relative sizes of the core and shell. For example, the Ag-Au core-shell structure exhibits a strong Ag surface plasmon resonance band combined with the ease of $\mathrm{Au}$ surface functionalization. ${ }^{1}(\mathrm{ZnS})-(\mathrm{CdSe})$ core- shell semiconductor materials show improved luminescence quantum yields. ${ }^{2}$ Bimagnetic $(\mathrm{FePt})-\left(\mathrm{Fe}_{3} \mathrm{O}_{4}\right)$ core-shell structures display strong exchange-spring behavior and an enhanced energy product. ${ }^{3}$ These physical and chemical properties of core-shell nanoparticles strongly depend on the structure of the core, the shell, and the interface. For magnetic nanoparticles, the biomedical applications of ferromagnetic iron oxide nanoparticles have been intensively studied due to their good biocompatibility. ${ }^{4}$ Recently, there is an increasing interest in developing alternative high moment metallic (Co, Fe, and related alloys) nanoparticles for specific biomedical applications that require significant generation of forces, such as bioseparation and targeted delivery. ${ }^{5}$ However, biological applications of cobalt-based nanoparticles are limited by their poor biocompatibility and resistance to oxidation. As a result, much effort has been made in creating a biocompatible interface for high magnetic moment cobalt nanoparticles by growing a gold shell. Development of such materials is expected to translate into useful and important biomedical/diagnostic applications with enhanced detection sensitivity. Gold-coated magnetic nanoparticles have been reported by different groups. ${ }^{6-10}$ However, most of the synthetic procedures were limited by their reproducibility and the control of shell uniformity and crystallinity because of the use of a strong reducing agent (borohydride) and an oxygen and water rich environment. These problems in synthesis can be partially

* To whom correspondence should be addressed. E-mail: kannanmk@ u.washington.edu.

University of Washington.

$\doteqdot$ ESFM-IPN.

$\S$ Current address: Center for Integrated Nanotechnology, Los Alamos National Laboratory, Los Alamos, NM 87544. solved by using a weak reducer such as hydrazine or a primary amine. ${ }^{11,12}$ Most recently, a more successful procedure was reported to create various core-shell structures utilizing a redoxtransmetalation process. ${ }^{13}$ In this method, an inorganic gold salt was transferred into nonpolar solvent using a phase-transfer agent to serve as a shell precursor. Subsequently, gold ions were reduced by cobalt due to the difference in their reduction potentials. One of the main issues in the redox-transmetalation process is the interface between the core and the shell because gaps or cavities are likely produced as the ions of the core materials diffuse out of the nanostructures. In fact, with use of cobalt nanoparticles as templates, gold hollow spheres with tunable optical properties have been successfully synthesized using the transmetalation method. ${ }^{14-16}$ Additionally, the evolution of magnetic properties in such displacement reactions can be a good indicator of the core-shell morphology. ${ }^{17}$ Besides the synthetic issues of core-shell nanostructures, it is also vital to properly characterize the core-shell structure of nanoparticles to confirm the core-shell morphology. Until now, microstructural analysis of core-shell nanostructure still remains a big challenge due to the small size of the grains and the complex crystal orientations. Here, we present the synthesis of $\mathrm{Co}-\mathrm{Au}$ core-shell nanoparticles using an organo-gold compound as precursor in a nonpolar solvent. Detailed structural analysis using high spatial resolution TEM techniques in conjunction with studies of their magnetic and optical properties provide convincing evidence for the core-shell morphology of the nanoparticles synthesized in our laboratory.

\section{Experimental Section}

Synthesis of Cobalt Nanoparticle Seeds. For the synthesis of $\mathrm{Co}-\mathrm{Au}$ core-shell nanoparticles, cobalt seeds were first fabricated. All the procedures were carried out under an argon atmosphere to prevent oxidation. Monodispersed $6 \mathrm{~nm}$ cobalt nanoparticles were synthesized utilizing a procedure similar to the one reported elsewhere. ${ }^{12,18}$ Specifically, $1.58 \mathrm{mmol}(0.54$ g) of cobalt carbonyl dissolved in $3 \mathrm{~mL}$ of 1,2-diclrorobenzene (DCB), forming a precursor stock solution. The stock solution

\subsection{1/jp066871y CCC: $\$ 37.00$ @ 2007 American Chemical Society} Published on Web 01/17/2007 
was then injected into pre-heated DCB $\left(180{ }^{\circ} \mathrm{C}\right)$ solution containing a mixture of $0.6 \mathrm{mmol}(0.2 \mathrm{~mL})$ of oleic acid and $1.1 \mathrm{mmol}(0.34 \mathrm{~mL})$ of trioctylamine (TOA) and refluxed for $15 \mathrm{~min}$.

Synthesis of Co-Au Core-Shell Nanoparticles. Two milligrams of cobalt seeds after washing with methanol was redispersed into $5 \mathrm{~mL}$ of toluene, forming a homogeneous dark black solution. The seed solution was then heated up to $95^{\circ} \mathrm{C}$ slowly while stirring under an argon atmosphere. Gold precursor stock solution was prepared inside the glovebox by dissolving $0.01 \mathrm{~g}$ of $\left[\left(\mathrm{C}_{6} \mathrm{H}_{5}\right)_{3} \mathrm{P}\right] \mathrm{AuCl}$ and $0.5 \mathrm{~mL}$ of oleylamine into $3 \mathrm{~mL}$ of toluene. Here, the amine served as both a reducing and capping agent. The gold precursor solution was then injected into the preheated $\left(95^{\circ} \mathrm{C}\right)$ toluene solution and kept at this temperature for an hour. A yellowish black solution was obtained, leading to the formation of $9 \mathrm{~nm} \mathrm{Co}-\mathrm{Au}$ core-shell nanoparticles. Subsequently, these core-shell nanoparticles were made water-soluble by functionalizing them with hydrophilic thiol-containing surfactants, 11-mercaptoundecanoic acid, through the specific binding between gold and thiols (Supporting Information).

Characterization of the Co-Au Core-Shell Nanoparticles. The crystal structure of cobalt seeds and the core-shell nanoparticles were examined in powder form with a Rigaku $12.5 \mathrm{~kW}$ rotating anode X-ray diffraction system. The detailed core-shell structures were investigated by a wide range of TEM analysis, including bright field imaging (Phillips CM 100 TEM), high-resolution TEM, EELS, and Z-contrast imaging (a $200 \mathrm{kV}$, FEI F20 UT Tecnai, transmission electron microscope with an in-column monochromator). Temperature and field-dependent magnetization measurements were performed on a superconducting quantum interference device (SQUID) magnetometer (Quantum Design MPMS SQUID Magnetometer). Optical properties of solution-form samples were investigated on similar size pure cobalt, pure gold, and $\mathrm{Co}-\mathrm{Au}$ core-shell nanoparticles by UV - visible spectrophotometery (Carry 550).

\section{Results and Discussions}

After synthesis, cobalt nanoparticles seeds were coated with a layer of surfactants, which make them soluble only in organic nonpolar solvents due to the hydrophobic nature of the hydrocarbon chains. To obtain uniform core-shell structures, several strategies were applied for the synthesis of $\mathrm{Co}-\mathrm{Au}$ core-shell nanoparticles. First, a nonpolar solvent (toluene) was chosen to facilitate the formation of a homogeneous seed solution. Second and more importantly, an organo-gold compound was used as a precursor with distinct positive attributes that include the following: (1) It has very good solubility in toluene, forming a homogeneous solution together with the seeds. Phase transformation could possibly bring water, ions, or other molecules into the system, which affects the magnetic properties of the seeds. (2) $\left[\left(\mathrm{C}_{6} \mathrm{H}_{5}\right)_{3} \mathrm{P}\right] \mathrm{AuCl}$ precursor exists as a stable complex in toluene, which cannot react directly with cobalt. (3) The weak reducer, oleyamine, can slowly reduce the gold precursor on cobalt seeds to form a shell since the heterogeneous nucleation requires less activation energy. Additionally, this procedure avoids the interface problem due to ion diffusion. All these considerations allowed the formation of core-shell structure to take place in a controlled manner. These $\mathrm{Co}-\mathrm{Au}$ core - shell nanoparticles in organic solution are stable up to several months without the observation of a cobalt oxide signal in X-ray diffraction measurements. However, their stabilities were reduced after transfer into aqueous solution, mainly owing to the reaction of cobalt and thiol groups; this

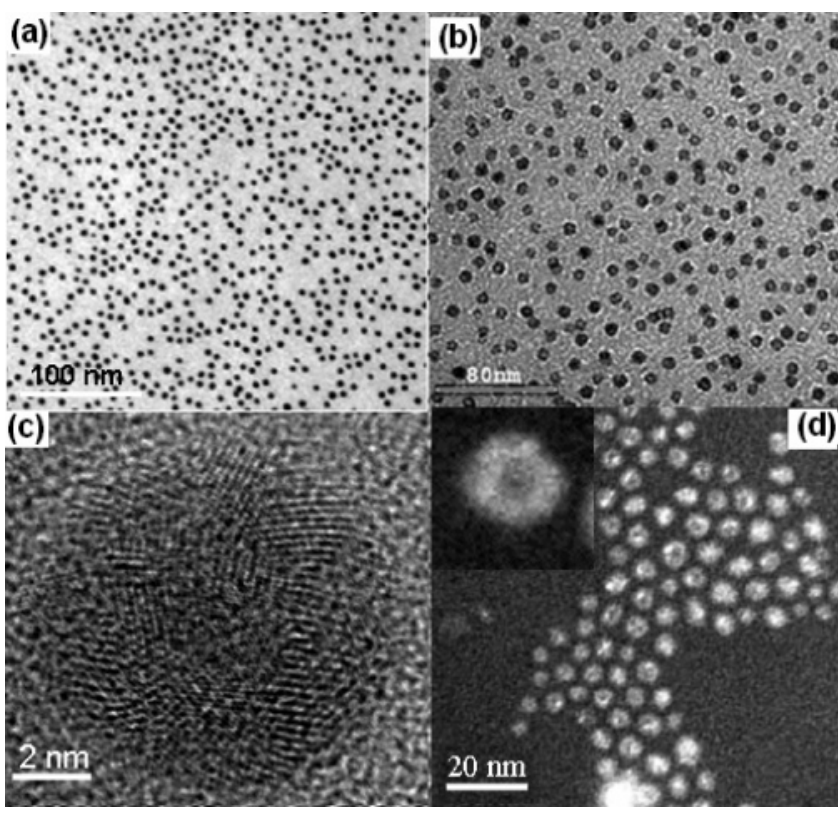

Figure 1. Nine nanometer $\mathrm{Co}-\mathrm{Au}$ core - shell nanoparticles: (a) cobalt nanoparticle seeds, (b) bright field TEM image, (c) high-resolution TEM image, and (d) Z-contrast image of $9 \mathrm{~nm} \mathrm{Co-Au} \mathrm{core-shell} \mathrm{nano-}$ particles shows the contrast difference between the relatively lighter shells and the darker centers, indicating the spatial distribution of a cobalt-rich core and gold-rich shell within the core-shell nanoparticles. A clearer Z-contrast image of a single core-shell nanoparticle is shown in the inset.

reaction pilled off gold grains from the structure. ${ }^{19}$ This assumption was also supported by the observation of a strong absorption around $505 \mathrm{~nm}$ in the UV-vis absorption spectra, which can be attributed to the absorption by small gold clusters (Supporting Information).

A bright field TEM image of the cobalt nanoparticle seeds is shown in Figure 1a, suggesting a narrow size distribution. Figure $1 \mathrm{~b}$ shows a representative bright field TEM image of 9 $\mathrm{nm} \mathrm{Co}-\mathrm{Au}$ core - shell nanoparticles. In principle, the contrast in a bright field TEM image has contributions from a mechanism based on both mass and thickness. The thicker and/or higher mass areas appear darker than thinner and/or lower mass region. The lighter cores and the darker shells of the TEM image suggest that the core-shell structure was formed. Cobalt has a lower atomic mass than gold; therefore, it is expected that a core made of cobalt should look lighter than the gold shell based on atomic mass contrast. Here, we believed that the atomic mass contribution was dominant over the thickness. The HRTEM image shows a single-crystal Co core surrounded by multiple gold grains, forming a raspberry structure. Clear $\mathrm{Co} / \mathrm{Au}$ boundaries are not observed due to the multiple gold grains on the cobalt surface. This structure suggests that gold has multiple nucleation sites on individual cobalt seeds during synthesis (Figure 1c). The core diameter is $5-6 \mathrm{~nm}$, close to the size of the cobalt seeds used during synthesis, and the shell is roughly $1.5-2.0$ $\mathrm{nm}$ thick. The HREM image clearly shows the structure of the shell, but the core is not well-resolved due to it being both in a different crystallographic orientation and embedded inside the thin shell. However, lattice spacing of 0.204 and $0.102 \mathrm{~nm}$, measured from the images of the shell combined with an inverse Fourier transform (IFT) analysis, corresponds to fcc Au (002) and (004) planes (Supporting Information). A Z-contrast image performed on these core-shell nanoparticles using scanning transmission electron microscopy ${ }^{20}$ is shown in Figure 1d. In this analysis, a high-intensity electron probe, typically sub- 

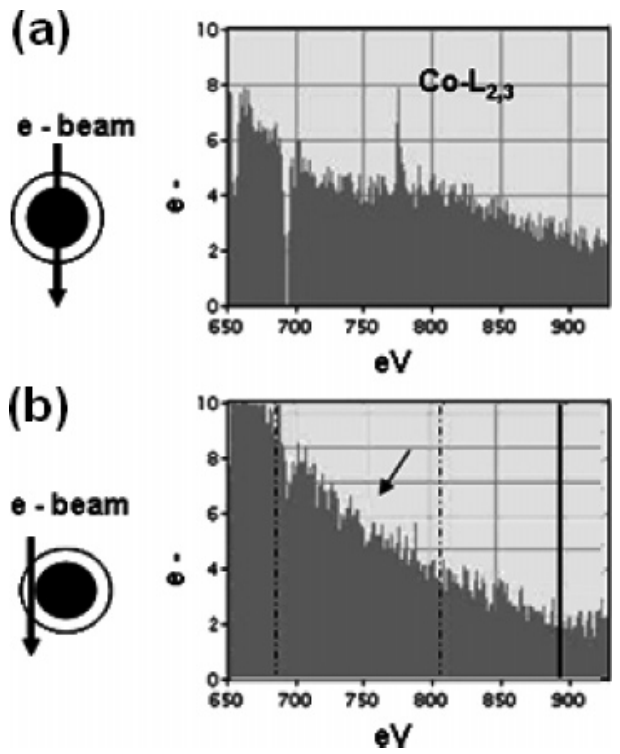

Figure 2. EELS spectra of $9 \mathrm{~nm}$ diameter $\mathrm{Co}-\mathrm{Au}$ core-shell nanoparticles from (a) center and (b) edge.

nanometer in diameter that determines the spatial resolution, is scanned across the sample and electrons incoherently scattered at high angles are detected by an annular detector. Element specificity is also a routine as the intensity of the scattered electrons is proportional to the square of the atomic number of the probed element. The contrast difference between the relatively lighter shells (higher atomic number) and the darker centers (lower atomic number) confirms the expected spatial distribution of cobalt and gold within the core-shell nanoparticles.

In addition to the Z-contrast imaging, the local chemistry was unequivocally determined by measuring the EEL spectra using a $1 \mathrm{~nm}$ focused probe. Figures $2 \mathrm{a}$ and $2 \mathrm{~b}$ show the EEL spectra focused on the core and the shell. The $\mathrm{L}_{3,2}$ edges in EELS of transition metals are marked by prominent features at the threshold, called "white lines", due to excitations from $2 \mathrm{p}_{3 / 2}\left(\mathrm{~L}_{3}\right)$ and $2 \mathrm{p}_{1 / 2}\left(\mathrm{~L}_{2}\right)$ spin-orbit split levels to the unoccupied $3 \mathrm{~d}$ states following the allowed dipole transitions. The observation of strong features at 779 and $794 \mathrm{eV}$ losses, corresponding to the Co $L_{2,3}$ edges in the EEL spectra, obtained when focused on the core, indicates the presence of cobalt; the absence of these edges when focused on the shell confirms the distinct chemical nature of the core and shell and suggests that the cobalt concentration in the shell is below the instrument detection limit of $\sim 5 \%$. Here, the Au core edge peak is not examined because its $\mathrm{Au} \mathrm{M} \mathrm{M}_{4,5}$ edges (2206 and $2291 \mathrm{eV}$ ) are outside the energy range $(<1000 \mathrm{eV})$ of facile EELS analysis in a TEM. This measurement was repeated several times with almost identical results.

In addition to the detailed TEM analysis, the crystal structure of these $\mathrm{Co}-\mathrm{Au}$ core-shell nanoparticles was studied by powder X-ray diffraction (XRD). The main peaks of the $\theta-2 \theta$ $\mathrm{X}$-ray scan were indexed as fcc gold crystal structure and the cobalt signal was barely seen (Figure 3a). Yet the crystal structure of pure cobalt seeds was indexed as the $\epsilon$-structure (Figure 3b). After being coated with gold grains, the cobalt signals are dominated by gold due to the much stronger absorption of $\mathrm{Cu} \mathrm{K} \alpha$ radiation by cobalt than gold. However, two more peaks were clearly seen from a detailed scan in a range of $40^{\circ}-50^{\circ}$, which matched the (310) and (311) peaks of $\epsilon$-cobalt (Figure 3a, inset); the main peak (221) of $\epsilon$-cobalt
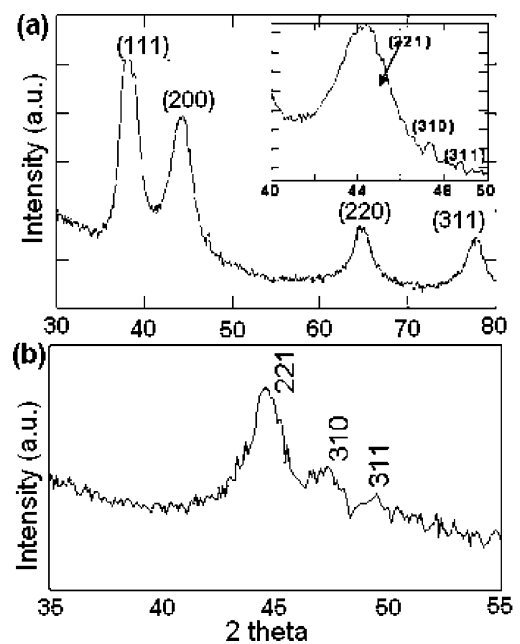

Figure 3. $\theta-2 \theta \mathrm{X}$-ray scans: (a) $9 \mathrm{~nm} \mathrm{Co}-\mathrm{Au}$ core-shell nanoparticles and the detailed scan in a narrow angular window is shown in the inset; (b) cobalt seeds.
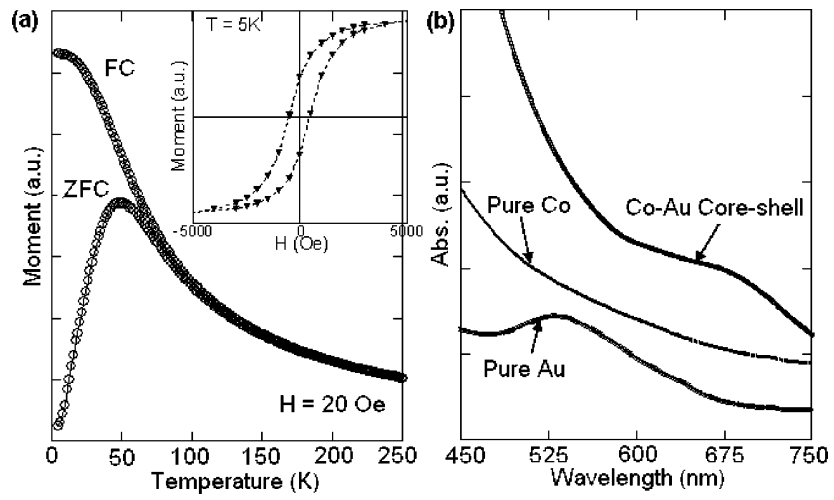

Figure 4. Nine nanometer $\mathrm{Co}-\mathrm{Au}$ core-shell nanoparticles: (a) ZFC/ FC magnetic measurements, inset $5 \mathrm{~K}$ hysteresis loop; (b) UV-visible spectra and the comparison with similar size pure cobalt and gold nanoparticles.

was not well-resolved since it overlaps with the (200) peak of fcc gold. Simulation of the XRD pattern was performed with JADE software, and the average crystalline size of these coreshell structures was determined by the Scherrer equation to be about $5 \mathrm{~nm}$ with $\pm 10 \%$ deviations (Supporting Information). This simulation demonstrated relative uniform particle size of these core-shell nanoparticles. The average crystalline size from the simulation was smaller than the size obtained from the TEM image, also suggesting the presence of multiple gold grains. The powder XRD analysis confirmed the presence of both cobalt and gold in large quantity.

Figure $4 \mathrm{a}$ shows the temperature-dependent magnetization measurements (zero field cooled and field cooled, ZFC/FC) of these core-shell nanoparticles using a SQUID magnetometer. The $\mathrm{ZFC} / \mathrm{FC}$ curves show a relative narrow peak around $55 \mathrm{~K}$, suggesting that these particles are superparamagnetic at room temperature and ferromagnetic below $55 \mathrm{~K}$. The latter was consistent with the observation of an open hysteresis loop at 5 $\mathrm{K}$ (Figure $4 \mathrm{a}$, inset). The magnetic size of the core-shell nanoparticle was estimated by comparing its blocking temperature with measurements on pure cobalt nanocrystals. This is done with an assumption that the gold grains do not affect the anisotropy constant of cobalt significantly due to the immiscible nature of cobalt and gold. For a given measurement time, $\tau$ of $100 \mathrm{~s}$, the blocking temperature $\left(T_{\mathrm{B}}\right)$ can be used to estimate 
the magnetic volume (eq 1). The observed $T_{\mathrm{B}} \sim 55 \mathrm{~K}$ corresponds to approximately $6 \mathrm{~nm}$ magnetic core.

$$
\tau=\tau_{0} \exp \left(\frac{K V}{k_{\mathrm{B}} T}\right) \Longrightarrow \frac{T_{\mathrm{B} 1}}{T_{\mathrm{B} 2}}=\frac{r_{1}{ }^{3}}{r_{2}{ }^{3}}
$$

In addition to the intrinsic magnetic properties of the cobalt core, the gold nanoshell brings in unique biocompatibility and near-infrared optical activity. The plasmon-derived optical resonance of gold shell can be dramatically shifted in wavelength from the visible region into infrared ${ }^{21}$ and the wavelength range spans the region of highest physiological transmissivity. ${ }^{22} \mathrm{UV}-$ visible spectra of our nanoparticles show a continuous decrease in intensity for pure Co nanoparticles and a distinct peak around $530 \mathrm{~nm}$ for pure Au nanoparticles. However, a pronounced peak around $680 \mathrm{~nm}$ was observed for the Au shell absorbance of these $\mathrm{Co}-\mathrm{Au}$ core-shell nanoparticles (Figure $4 \mathrm{~b}$ ). This red shift behavior of these core-shell nanoparticles is attributed to surface plasmon resonance enhanced absorption. It is expected to be similar to the one reported in the literature for a goldcoated dielectric core ${ }^{22}$ and depend on the relative size of the core and shell. Theoretical calculation on $\mathrm{Ag}-\mathrm{Au}$ core-shell structure also shows a red shift for gold-coated metallic cores. ${ }^{23}$ The red shift in wavelength of gold surface plasmon has also been observed experimentally in other bimetallic systems with a metal core as well, such as $\mathrm{Fe}-\mathrm{Au}$ core- shell nanoparticles. ${ }^{6}$

\section{Conclusions}

In conclusion, we have synthesized $\mathrm{Co}-\mathrm{Au}$ core-shell nanoparticles in a nonpolar environment and, using a range of complementary electron-optical characterization methods, provided a detailed analysis to confirm the formation of a cobalt core and a gold shell. The magnetic and optical properties are also consistent with this core-shell morphology; further, they may both be tuned by tailoring the size of the core and shell. The gold shell also provides a variety of surface functionalization opportunities. These tailorable magnetic/optical properties and ease of surface functionalization in these well-characterized core-shell nanostructures make them attractive candidates for a wide range of biomedical applications, such as separation, detection, and sensing.

Acknowledgment. This work was supported by NSF/DMR DMR-0501421 and the Campbell Endowment at UW. We thank PNNL and NCEM/LBNL for use of the TEM facilities.
Supporting Information Available: The inverse Fourier transform (IFT) analysis of the gold shell and Jade software simulation results of $\mathrm{X}$-ray diffraction pattern for $\mathrm{Co}-\mathrm{Au}$ coreshell nanoparticles. This material is available free of charge via the Internet at http://pubs.acs.org.

\section{References and Notes}

(1) Cao, Y.; Jin, R.; Mirkin, C. A. J. Am. Chem. Soc. 2001, 123, 1961

(2) Kortan, A. R.; Hull, R.; Oplia, R. L.; Bawendi, M. G.; Steigerwald, M. L.; Carrol, P. J.; Brus, L. E. J. Am. Chem. Soc. 1990, 112, 1327. 4,187

(3) Zeng, H.; Li, J.; Wang, Z. L.; Liu, J. P.; Sun, S. Nano Lett. 2004

(4) Tartaj, P.; del Puerto Morales, M.; Veintemillas-Verdaguer, S.; González-Carreño, T.; Serna, C. J. J. Phys. D: Appl. Phys. 2003, 36, R182.

(5) Chen, M.; Yamamuro, S.; Farrell, D.; Majetich, S. A. J. Appl. Phys. 2003, 93, 7551 .

(6) Zhou, W.; Kumbhar, A.; Wiemann, J.; Fang, J.; Carpenter, E. E.; O'Conner, C. J. J. Solid State Chem. 2001, 159, 26.

(7) Cho, S.-J.; Kauzlarich, S. M.; Olamit, J.; Liu, K.; Grandjean, F.; Rebboulh, L.; Long, G. J. J. Appl. Phys. 2004, 95, 6804.

(8) Lyon, J. L.; Fleming, D. A.; Stone, M. B.; Schiffer, P.; Williams, M. E. Nano Lett. 2004, 4, 719

(9) Cushing, B. L.; Golub, V.; O’Connor, C. J. J. Phys. Chem. Solids 2004, 65, 825.

(10) Cho, S.-J.; Idrobo, J.-C.; Olamit, J.; Liu, K.; Browning, N. D.; Kauzlarich, S. M. Chem. Mater. 2005, 17, 3181.

(11) Bao, Y.; Krishnan, K. M. J. Magn. Magn. Mater. 2005, 293, 15.

(12) Bao, Y.; Pakhomov, A. B.; Krishnan, K. M. J. Appl. Phys. 2005, 97, 10J317.

(13) Lee, W.; Kim, M. J.; Choi, J.; Park, J.-II.; Ko, S. J.; Oh, S. J.; Cheon, J. J. Am. Chem. Soc. 2005, 127, 16090.

(14) Liang, H. P.; Wan, L. J.; Bai, C. L.; Jiang, L. J. Phys. Chem. B 2005, 109, 7795 .

(15) Schwartzberg, A. D.; Olson, T. Y.; Talley, C. E.; Zhang, J. Z. J. Phys. Chem. B 2006, 110, 19935.

(16) Linag, H. P.; Guo, Y. G.; Zhang, H. L.; Hu, J. S.; Wan, L. J.; Bai, C. L. Chem. Commun. 2004, 1496.

(17) Mandal, S.; Krishnan, K. M. J. Mater. Chem. 2007, DOI: 10.1039/ b613371c

(18) Puntes, V. F.; Krishnan, K. M.; Alivisatos, P. A. Science 2001, 291,2115

(19) Yin, Y.; Rioux, R. M.; Erdonmes, C. K.; Hughes, S.; Somorjai, G. A.; Alivisatos, P. A. Science 2004, 304, 714.

(20) Keyse, R. J.; Garrett-Reed, A. J.; Goodhew, P. J.; Lorimer, G. W. Introduction to Scanning Transmission Electron Microscopy; BIOScientific Publisher, Springer: New York, 1998; Chapter 7.

(21) Hao, E.; Li, S.; Bailey, R. C.; Zou, S.; Schatz, G. C.; Hupp, J. T. J. Phys. Chem. B 2004, 108, 1224.

(22) Oldenburg, S. J.; Averitt, R. D.; Westcott, S. L. Chem. Phys. Lett. 1998, 288, 243.

(23) Kreibig, U.; Vollmer, M. Optical Properties of Metal Clusters; Springer: Berlin, 1995; Chapter 4, p 373. 